\title{
PC_Eyewitness: Evaluating the New Jersey method
}

\author{
Otto H. Maclin and Colin M. Phelan \\ University of Northern Iowa, Cedar Falls, Iowa
}

\begin{abstract}
One important variable in eyewitness identification research is lineup administration procedure. Lineups administered sequentially (one at a time) have been shown to reduce the number of false identifications in comparison with those administered simultaneously (all at once). As a result, some policymakers have adopted sequential administration. However, they have made slight changes to the method used in psychology laboratories. Eyewitnesses in the field are allowed to take multiple passes through a lineup, whereas participants in the laboratory are allowed only one pass. PC_Eyewitness (PCE) is a computerized system used to construct and administer simultaneous or sequential lineups in both the laboratory and the field. It is currently being used in laboratories investigating eyewitness identification in the United States, Canada, and abroad. A modified version of PCE is also being developed for a local police department. We developed a new module for PCE, the New Jersey module, to examine the effects of a second pass. We found that the sequential advantage was eliminated when the participants were allowed to view the lineup a second time. The New Jersey module, and steps we are taking to improve on the module, are presented here and are being made available to the research and law enforcement communities.
\end{abstract}

When a crime occurs, police attempt to solve it by gathering evidence directing them to a suspect. This evidence may later be used to convict the suspect in a court of law. One type of evidence that is often presented in court trials is an identification made by an eyewitness. Eyewitnesses to criminal acts are potentially powerful resources for "serving justice" to those who violate the laws of society. Unfortunately, eyewitness identifications are not always accurate (Wells et al., 1998). When eyewitnesses erroneously identify innocent suspects, there is a risk that the latter may be convicted of crimes they did not commit. In fact, mistaken eyewitness identification has been shown to be the primary factor responsible for the wrongful conviction of innocent individuals recently exonerated through DNA evidence (Connors, Lundregan, Miller, \& McEwan, 1996; Scheck, Neufeld, \& Dwyer, 2000). It is bad enough to falsely convict a person; the other problem is that the guilty person may still be free to commit more crimes. Given that eyewitness identification is so important, it is crucial that those who conduct research on this topic examine how people function as eyewitnesses under various circumstances, and in particular, how those circumstances affect identification accuracy.

Specifically, many researchers have directed their attention toward examining the advantages and disadvantages of different lineup administration procedures used by police. The traditional procedure is to present all of the members of the lineup at the same time (i.e., simultaneously). For example, in order to administer a six-member lineup simultaneously, police would present all six members at once to the eyewitness (typically in a $3 \times 2$ photo array commonly known as a "six-pack"). The witness would either identify one of the members as the perpetrator or reject the lineup by indicating that the perpetrator was not in the lineup. Research has found that people tend to make more mistakes when a simultaneous lineup does not contain the perpetrator (referred to as "target absent") than when the perpetrator is present in the lineup (referred to as "target present"). Different mistakes carry different consequences. A mistake in the target-absent lineup can result in a false alarm, directing the investigation toward an innocent suspect. A mistake in the target-present lineup can result in rejecting the lineup, thus potentially directing the investigation away from the actual perpetrator.

Researchers sought an alternative lineup administration procedure that would reduce the number of false alarms without reducing the number of hits. They found that when photos were shown one at a time (sequentially), and witnesses were asked to determine for each face shown whether or not it was a photo of the perpetrator, false alarms were reduced (Lindsay \& Wells, 1985). It has been argued that different judgment processes may be used in the lineup decision, depending on whether eyewitnesses are viewing a simultaneous or a sequential lineup. Wells (1984) proposed that individuals viewing a simultaneous lineup tend to use a relative judgment process (viewing the lineup in its entirety and comparing all of the members relative to one another) before making a lineup decision.

O.H. MacLin, otto.maclin@uni.edu 
They would then choose the lineup member who most closely matched their memory of the perpetrator, relative to other lineup members. This type of behavior can result in increased false alarms in the target-absent condition.

Lindsay and Wells (1985) argued that individuals viewing a sequential lineup tend to use an absolute judgment process, meaning that they compare each lineup member individually to their memory of the perpetrator, as opposed to viewing the lineup as a whole. Each member of a sequential lineup is presented by himself, and eyewitnesses must make a decision for each member individually ("Is this the perpetrator-yes or no?").

A meta-analysis conducted by Steblay, Dysart, Fulero, and Lindsay (2001) examined studies comparing the two lineup administration procedures and found that although simultaneous lineup administration produced greater accuracy for target-present lineups (i.e., a higher rate of correct identifications), sequential administration produced greater accuracy for target-absent lineups (i.e., a higher rate of correct rejections). The finding that sequential administration reduces the rate of false identification in comparison with simultaneous administration has been referred to as the "sequential superiority effect."

Although the police have typically used the traditional simultaneous procedure (in part because of its ease of administration), there is currently a movement toward use of the sequential procedure. Some policymakers have begun to adopt the use of sequential administration in the field (Farmer, 2001). There are, however, trade-offs with the decision to adopt sequential administration for use in reallife criminal situations. First, there have been no published findings indicating that the "sequential superiority effect" has been found in the field. Second, the sequential procedure that has been implemented in the field is not identical to the sequential procedure used in the research laboratory, where the benefits were first seen.

Sequential procedures in the laboratory typically lead participants to believe that they will see more lineup members than are actually presented to them, a technique that has been referred to as "backloading" (MacLin \& Malpass, 2005). For example, participants may be given an answer sheet with 12 lines, presumably representing 12 lineup members to be viewed, yet only 6 members may actually be presented in the lineup. Backloading is used to reduce the likelihood of an identification increasing as the lineup is coming to a finish, and may also create a criterion shift, causing participants to become more conservative about their decision, in case a "better" photo is presented.

Another difference in the laboratory studies is that participants are typically allowed to view a sequential lineup only once, and the lineup is terminated after the first "Yes" response. If all of the photos are viewed, and they all receive a "No" response, this would indicate that the participant had rejected the lineup. In the lab, participants are unable to go back to start the lineup over.

In the field, the sequential procedure has succumbed to subtle modifications not yet studied in the laboratory. The states of New Jersey (Farmer, 2001) and Minnesota (Turtle $\&$ Steblay, 2005) have adopted sequential procedures allow- ing eyewitnesses to view the lineup a second time after they make an initial pass. In the field, the lineup does not terminate on a "Yes" response; witnesses are allowed to continue the lineup and are allowed to make multiple passes, regardless of how many times they may make a "Yes" or "No" response during the sequential presentation.

Most, if not all, of the lineup administration in the field is done "by hand," and may be subject to administrator bias. Computerized programs, such as PC_Eyewitness (PCE), have been developed to administer lineups in the laboratory (MacLin, Meissner, \& Zimmerman, 2005). Computerized administration using PCE has been demonstrated to be similar to the traditional paperand-pencil testing that much of the eyewitness identification research data are based on (MacLin, Zimmerman, \& Malpass, 2005). Computerized lineup administration systems for the field have been recommended by the American Bar Association (Taslitz, 2004), and are under development in our laboratory. We are currently working with the Waterloo (Iowa) Police Department to develop a lineup construction and administration program based on PCE, which will allow the investigators to administer sequential lineups with a desktop computer using a Windows operating system. However, we must continually evaluate the design changes that occur when the procedures are transferred from the laboratory to the field, as mentioned earlier. To do this, we developed a new module for PCE, referred to as the New Jersey module. Like PCE, the New Jersey module was written in Visual Basic 6.0 (although the system we are developing for the field is being written in Visual Basic Express 2005). Details about the New Jersey module are provided in the Method section.

The New Jersey module was designed to explore the issue of whether or not the "sequential superiority effect" would be found when the New Jersey method was used for lineup administration. Specifically, would there still be a lower rate of false identifications for the sequential procedure (in comparison with the traditional simultaneous procedure) when participants took a second pass through the lineup? Or would the data become more similar to those found when using simultaneous lineups? Based on the Steblay et al. (2001) meta-analysis, we hypothesized that the first pass on the sequential lineup would produce fewer correct identifications and more correct rejections relative to the simultaneous condition. However, in the second pass, because participants would know the actual number of lineup members and because they would have had the chance to compare the lineup members with one another, we hypothesized that there would be no difference in lineup performance across the three conditions. We also hypothesized that there would be no significant differences in the data obtained from the second pass, regardless of whether they were collected from a simultaneous or a sequential lineup.

\section{METHOD}

\section{Participants}

A total of 230 students at a Midwestern university participated in this experiment for partial course credit. The median age of the 
participants was 18 (ranging from 18 to 25 years), with females accounting for $82.6 \%$ of the participants.

\section{Materials}

Staged crime video. A single video clip with a duration of about 3 min, which depicted the theft of a calculator from a library, was used to display the male perpetrator, who was visible during the entire scene.

Lineups. A target-present lineup was constructed based on the single perpetrator. It included a full-frontal photo of the perpetrator seen in the video, as well as five photos of other males matching the modal description of the perpetrator (e.g., match to description). These lineup members would be known to be innocent, in an actual police lineup, and would act as fillers, or foils. A second lineup was created with the perpetrator absent from the lineup (target absent). In the target-absent lineup, the photo of the perpetrator was replaced by a photo of an "innocent suspect" closely matching the description of the perpetrator. For both the simultaneous and the sequential lineups, the positions of the perpetrator and the foils were randomized for each participant.

Apparatus. PCE, a computer program developed to present stimuli on monitors and collect participant responses in eyewitness identification research, was used. PCE includes an icon-based design mode supporting 11 event modules that can be sequenced on the basis of the researcher's needs (MacLin, Meissner, \& Zimmerman, 2005). After the events have been sequenced, relevant parameters can be set and stored in a control file. The control file can be accessed later, when the experiment is administered. In administration mode, PCE generates an output file detailing the input file used for the session, the series of events presented, and each response generated by the participant. For more details about PCE, including a Web address to the program's tutorial and download site, see MacLin, Meissner, and Zimmerman.

New Jersey module overview. We designed a 12 th event module for PCE, to examine the New Jersey method; it is currently available for use with PCE. The New Jersey module is similar to the basic sequential lineup module, in that the administrator selects up to 12 photos to be used in the lineup, determines which photo belongs to the suspect, and selects the type of randomization to be used. The administrator can select: (1) no randomization, (2) full randomization, (3) partial randomization disallowing the suspect to be placed in Position 1, and (4) randomization of the foils only. The administrator can determine the number of passes the participant is allowed to take, or the administrator can require that multiple passes be taken. Instructions to the participant regarding his or her choice to take a second pass can be changed. For example, we have considered changing the language from "Do you want to take a second pass?" to "Do you need to take a second pass?" to reduce the number of passes the participant takes. Finally, the administrator can control the duration of the changeover delay.

For our study, the participants were required to view the lineup a second time (with the instruction "Do you need to take a second pass?"). The computer displayed the lineup a second time, with the photos positioned in the original sequence. We designed a second experimental module (not offered with PCE) to present a sequential lineup on the first pass and then present a simultaneous lineup if a second pass is requested. The second-pass simultaneous lineup was identical in structure to the simultaneous lineup displayed in the simultaneous-only comparison condition. Other PCE event modules used in this experiment include the instructions, video, distractor task, open-ended response, scale, and demographics.

\section{Design}

The design used was a 3 (lineup administration procedure: simultaneous, sequential/sequential, or sequential/simultaneous) $\times 2$ (lineup type: target present or target absent). Because participants were allowed to view the simultaneous lineup as long as they wanted to, an opportunity to view the lineup a second time made little sense. Therefore, the simultaneous lineup was used as a comparison with the first pass on the sequential lineup and separately as a comparison with the data collected in the second pass. Because multiple "Yes" responses can be made during each pass, we used the first "Yes" response in each pass as the identification. This is consistent with previous research using sequential administration (Steblay et al., 2001). The dependent measure was accuracy of the lineup identification.

\section{Procedure}

Sessions were conducted with 10 or 12 participants at a time, who were assigned to individual computer stations. They were instructed that the experiment was self-paced and that they should follow the instructions presented on the computer screen. The participants were randomly assigned to experimental conditions. The instructions explained that they would be viewing a video clip and that they should pay close attention because they would later be asked questions about the video. Specifically, "We are going to ask you to watch a short video clip. Please pay attention, as you will be asked questions regarding the video. We will explain the purpose of the study once we have finished."

The participants were next asked to solve simple math problems for $15 \mathrm{~min}$ (a distractor task). The instructions for the math task were as follows: "Next we will ask that you spend a few minutes solving math problems." The participants were then asked to provide a verbal description of the perpetrator by typing their response into a text box. The instructions were:

Next, you will be asked to describe in as much detail as possible the perpetrator you saw in the video. Use the text box that will be provided to write the details about what the perpetrator's FACE looked like. You should attempt to describe the person in sufficient detail such that someone else could identify him on the basis of the description you provide. As describing a face is often a difficult task, it is important that you concentrate and stay focused for the next few minutes. Remember: You should attempt to describe the person in sufficient detail such that someone else could identify him on the basis of the description. As describing a face is often a difficult task, it is important that you concentrate and stay focused for the next few minutes.

They were then asked to evaluate how well they thought they would do on a lineup task, using a scale from 1 (not very well) to 9 (very well ). Next, they were instructed that they would be seeing a lineup that might or might not contain the perpetrator. The positions of the lineup members were randomized by PCE for each participant. Instructions for the simultaneous identification task were as follows:

In a moment you will be given a lineup containing six faces. Your task is to select the perpetrator from the video that was shown to you at the beginning of the experiment; however, this person MAY Or MAY NOT be present in the lineup. Be aware that the individual may be wearing different clothing and may look somewhat different than in the video. If you are absolutely sure that none of the six faces is familiar to you, please click on the button that says, 'I DON'T SEE THE FACE.' Otherwise, type the number in the text box corresponding to the face that you think you saw as the assailant in the video.

The instructions for the sequential presentation were similar to those for the simultaneous presentation, and were as follows:

In a moment you will be given a lineup of a series of faces. Your task is to select the perpetrator from the video that was shown to you at the beginning of the experiment; however, this person MAY Or MAY NOT be present in the lineup. Be aware that the individual may be wearing different clothing and may look somewhat different than in the video. You will view each face one at a time. If you are absolutely sure that the face is familiar to you, please click on the button that says, "YES." Otherwise, click on "NO" if the face is not familiar to you.

The participants were then asked a series of questions, which did not affect the outcome of the lineup decisions and will be reported 
elsewhere. They then completed a demographics questionnaire, providing their age, sex, race, and current year in college. Finally, they were thanked and informed that no actual theft occurred, that the people they saw in the video were actors, and that no one was in trouble.

The participants assigned to the simultaneous condition were presented with a six-member $(2 \times 3)$ photo array with the position number of the lineup member located directly below the photo. They were instructed to indicate whether or not the perpetrator was present by entering the position number in a text box located near the bottom right of the monitor. If they did not think the perpetrator was present, they were instructed to reject the lineup by clicking on a button located near the bottom right of the screen indicating that they did not see the perpetrator.

The participants assigned to the sequential conditions were presented with the photos one at a time. The photos in the sequential lineups were of the same dimensions as those displayed in the simultaneous condition. The participants were instructed to indicate whether or not the photo belonged to the perpetrator by clicking on either a "Yes" or a "No" button located directly below the photo. When the participant made a response, the response was recorded and the next photo in the sequence was displayed, replacing the previous photo. When all six photos had been displayed, the participant was instructed that he or she would have a chance to see the lineup again. The original positions of the lineup members were maintained in the second pass. The participants were presented with either a sequential lineup or a simultaneous lineup, depending on the experimental condition they were assigned to at the beginning of the study.

\section{RESULTS}

\section{Lineup Performance: First Pass}

Target-present lineups. When using the targetpresent lineups, there are three classes of responses that participants can make. They can make a correct identification by identifying the perpetrator seen in the video, they can make an incorrect identification by identifying a lineup member known to be innocent (i.e., a foil), or they can incorrectly reject the lineup by not identifying any lineup member. In the simultaneous condition, $47.9 \%$ of the participants correctly identified the perpetrator, whereas $27.1 \%$ rejected the lineup and $25 \%$ identified a foil (see Table 1). In the sequential/sequential condition, $24.2 \%$ of the participants correctly identified the perpetrator, whereas $72.7 \%$ rejected the lineup and $3.1 \%$ identified a foil. In the sequential/simultaneous condition, $21.7 \%$ of the participants correctly identified the perpetrator, whereas $67.4 \%$ rejected the lineup and $10.9 \%$ identified a foil. The higher rate of correct identifications found for simultaneous administration was significant $\left[\chi^{2}(126)=\right.$ 9.69, $p=.002]$.

A closer examination of the specific lineup responses (correct identification, foil identifications, and lineup rejections) for the target-present lineups follows. Correct identifications for the simultaneous-only administration were significantly higher than those for either of the sequential lineup presentations $\left[\chi^{2}(41)=82, p=.001\right]$. Foil identifications for the simultaneous lineups were significantly higher than those for either of the sequential lineup presentations $\left[\chi^{2}(18)=36, p=.001\right]$. Finally, lineup rejections were higher for the two sequential lineup types relative to the simultaneous lineup $\left[\chi^{2}(68)=136, p=.001\right]$.

Target-absent lineups. When using the target-absent lineups, there are three classes of responses that participants can make. They can make a correct rejection by not identifying anyone from the lineup, they can make a false identification by identifying the innocent suspect, or they can make an incorrect identification by identifying a foil.

In the simultaneous condition, $50 \%$ of the participants correctly rejected the lineup, whereas $50 \%$ identified the innocent suspect. In the sequential/sequential condition, $100 \%$ of the participants correctly rejected the lineup. In the sequential/simultaneous condition, $82.9 \%$ of the participants correctly rejected the lineup, whereas $17.1 \%$ identified the innocent suspect (see Table 1). The higher rate of correct rejections found for sequential administrations was significant $\left[\chi^{2}(130)=20.67, p=.000\right]$.

\section{Lineup Performance: Second Pass}

Only participants in the sequential lineup condition were given a second pass.

Target-present lineups. In the sequential/sequential condition, $39.4 \%$ of the participants correctly identified the perpetrator, whereas $48.5 \%$ rejected the lineup and $12.1 \%$ identified a foil. In the sequential/simultaneous condition, $50 \%$ of the participants correctly identified the perpetrator, whereas $30.4 \%$ rejected the lineup and $19.6 \%$ identified a foil (see Table 1). No significant differences were found between simultaneous and sequential presentation in the second pass for the rate of correct identifications $\left[\chi^{2}(79)=.871, p=.351\right]$.

In the first pass, an advantage for simultaneous administration with regard to correct identification rate $(50 \%)$ was found. In comparison with the correct identification rate for the second pass in the sequential/sequential condition,

Table 1

\begin{tabular}{|c|c|c|c|c|c|c|}
\hline & \multicolumn{2}{|c|}{ Simultaneous Only } & \multicolumn{2}{|c|}{ Sequential/Simultaneous } & \multicolumn{2}{|c|}{ Sequential/Sequential } \\
\hline & 1st Pass & 2nd Pass & 1st Pass & 2nd Pass & 1st Pass & 2nd Pass \\
\hline Target Present & $n=48$ & & $n=46$ & & $n=33$ & \\
\hline Correct identification & 47.9 & & 21.7 & 50 & 24.2 & 39.4 \\
\hline Foil identification & 25 & & 10.9 & 19.6 & 3.1 & 12.1 \\
\hline Reject lineup & 27.1 & & 67.4 & 30.4 & 72.7 & 48.5 \\
\hline Target Absent & $n=30$ & & $n=41$ & & $n=32$ & \\
\hline Correct rejection & 50 & & 82.9 & 43.9 & 100 & 40.6 \\
\hline False identification & 50 & & 17.1 & 36.6 & & 34.4 \\
\hline Foil identification & & & & 19.5 & & 25 \\
\hline
\end{tabular}

Note-The data reported are percentages. 
the simultaneous condition advantage is lost $\left[\chi^{2}(81)=\right.$ $.575, p=.448]$. In comparison with the correct identification rate for the second pass in the sequential/simultaneous condition, the simultaneous condition advantage was reduced $\left[\chi^{2}(94)=.041, p=.840\right]$.

Target-absent lineups. In the sequential/sequential condition, $40.6 \%$ of the participants correctly rejected the lineup, whereas $34.4 \%$ identified the innocent suspect and $25 \%$ identified a foil. In the sequential/simultaneous condition, $43.9 \%$ of the participants correctly rejected the lineup, whereas $36.6 \%$ identified the innocent suspect and $19.5 \%$ identified a foil (see Table 1). No significant differences were found between simultaneous or sequential presentation in the second pass for the rate of correct rejections $\left[\chi^{2}(73)=.038, p=.845\right]$.

In the first pass, there was an advantage for sequential administration with regard to correct rejection rate $(100 \%)$. However, in comparison with the correct rejection rate for the simultaneous condition (50\%), the advantage of sequential administration in the first pass was lost when the second pass was administered either sequentially $\left[\chi^{2}(62)=.036, p=.851\right]$ or simultaneously $\left[\chi^{2}(71)=\right.$ $.000, p=.994]$.

\section{DISCUSSION}

Eyewitness identification is an important source of evidence, and is studied by psychologists interested in reducing false convictions. It has been demonstrated that sequential administration of lineups reduces the number of false identifications, but also reduces the number of correct identifications, to some extent (Steblay et al., 2001). Guidelines based on eyewitness identification research have been proposed by the federal government (Technical Working Group for Eyewitness Evidence, 2003), and law enforcement has begun to follow these guidelines (Farmer, 2001). However, as policy is implemented, compromises, such as allowing a second pass, can be introduced. It is important for eyewitness researchers to examine the changed procedures to determine their efficacy.

The computer program PCE (MacLin, Meissner, \& Zimmerman, 2005) has been used to collect data in several eyewitness laboratories across the United States, Canada, the United Kingdom, and South Africa. It has been demonstrated as a useful tool in collecting eyewitness identification data (MacLin, Zimmerman, \& Malpass, 2005) and it is currently being modified for use in constructing and administering lineups in the field (MacLin \& Meissner, 2005). In this article, we presented data from a new PCE module designed to administer lineups in a way similar to the procedures used in New Jersey and Minnesota. Specifically, the New Jersey module allows participants to view the lineup a second time.

We found that presenting the lineup a second time significantly reduces any advantage found in the sequential administration, regardless of whether the second pass was presented sequentially or simultaneously. This is potentially problematic for those promoting the sequential administration of lineups (Lindsay, 1999); however, it should be noted that even with the second pass, performance was never worse than that found in simultaneous administration. Also, this is of course only one study examining lineup performance using a second pass. We hope that by providing the New Jersey module to the research community, we can encourage more researchers to examine the administration of sequential lineups in the field.

Why the loss of the superiority effect? There are two benefits of the sequential lineup relative to the simultaneous lineup. The first is that participants are not able to compare the photos in the lineup directly with one another when the photos are viewed one at a time, but they are able to make these comparisons when the photos are administered simultaneously. Wells (1993) demonstrated that participants appear to be making relative judgments when photos are administered simultaneously, and he posited that participants who are administered photos sequentially make absolute judgments, comparing the photos to their memory of the perpetrator, rather than to each other. When a second pass is taken, participants are more familiar with the lineup members and may begin making lineup decisions by comparing the photos to one another, as they would in the simultaneous lineups. There is reason to believe that this is happening, because identification accuracy in the second pass is not significantly different between the simultaneous and sequential administrations.

The second benefit of the sequential lineup that is lost with the second pass is that the participant now has a good idea of the number of photos in the lineup. Eyewitness identification studies in the laboratory have used a technique referred to as backloading, whereby participants are led to believe that they will be seeing more photos than are actually in the lineup. For example, participants are often given answer sheets with up to 12 places to make responses, when in fact they were shown only six photos. With the simultaneous lineup, it is obvious that six photos are being viewed. When participants are allowed a second pass, the benefit of backloading is reduced.

It is also important to note that in the laboratory experiments, the sequential lineup typically terminates with a "Yes" response. This places the participant in a position to be more conservative about his or her decisions, effectively creating a criterion shift (Meissner, Tredoux, Parker, \& MacLin, 2005). In the field, the witness is allowed to make "Yes" responses and still view the remaining lineup members.

Although it is too early to determine exactly why the superiority effect is lost, there are also benefits gained with the sequential lineup. First, and possibly most importantly, law enforcement is beginning to adopt our recommendations. Second, law enforcement is beginning to experiment with using computers to administer lineups (e.g., MacLin \& Meissner, 2005). With computerized administration, the lineups are administered double-blind, which can reduce administrator bias by preventing any unconscious "tells" provided by the administrator, who may know who the perpetrator is (Wells, 1993; Wells et al., 2000).

It should be noted that we forced the participants to take a second pass through the lineup. It is possible that not all of the participants would have taken a second pass, given the option not to. We are currently examining im- 
provements and add-ons to the New Jersey method. For instance, we are currently allowing participants unlimited passes through the sequential lineups. We hypothesize that taking additional passes through the lineup may be diagnostic of the participants' memory quality; participants with a poor memory might require several additional passes through the lineup. We are also experimenting with adding a changeover delay. Changeover delays have been demonstrated to reduce rapid switching between alternative response options (Shull \& Pliskoff, 1967). It is possible that some participants might take a second pass, given the option, simply out of curiosity. We have added a condition in which participants are required to wait a period of time before being allowed a second pass. In this manner, they may be discouraged from taking a second pass. It is likely that the changeover delay will reduce the number of participants viewing the lineup more than once, with only those who require a second pass taking one. We are also experimenting with the response buttons. Currently, participants are required to make a "Yes" or "No" response for each face they view. It is likely that participants are not always able to make these distinct categorizations. Participants might be unsure about some of the photos. We are currently examining the effect of allowing participants to indicate that they are not sure, by adding a third choice. Participants will be able to indicate "Yes," "No," or "Not Sure." Finally, we are examining the effect of randomizing the lineup members in each pass. If participants are memorizing aspects of the lineup and using this information in the second pass, this randomization should require the participants to remember the particular photos rather than the position numbers. For example, this would prevent participants from saying something like "Number 2 and Number 6 look a lot like the perpetrator."

As our science advances our understanding of eyewitness identification, some of the advances make their way into policy and are administered in the field. Compromises are inherent in policy, and these compromises (such as allowing a second pass) may or may not affect the results found in the laboratory. It is therefore critical that we examine these changes, regardless of how subtle or seemingly innocuous they may appear. In this manner, the applied research in eyewitness identification coevolves with changes in the field. As this occurs, laboratories such as ours take on a research and development role with law enforcement. This is an important role, because most police departments do not have the funding or facilities to conduct their own research. Finally, we should note that the data from this study were collected on the basis of a single video containing a perpetrator, and that two lineups were created (a target-present lineup and a target-absent lineup) based on the perpetrator. Although this may limit the generalizability of these results (Wells \& Windschitl, 1999), we believe that the data are of sufficient caliber to illustrate and illuminate the phenomenon examined here.

\section{AUTHOR NOTE}

Correspondence concerning this article should be addressed to $\mathrm{O} . \mathrm{H}$. MacLin, Department of Psychology, University of Northern Iowa, Cedar Falls, IA 50614 (e-mail: otto.maclin@uni.edu).

\section{REFERENCES}

Connors, E., Lundregan, T., Miller, N., \& McEwan, T. (1996). Convicted by juries, exonerated by science: Case studies in the use of DNA evidence to establish innocence after trial. Alexandria, VA: National Institute of Justice.

FARMER, J. J., JR. (2001, April 18). Attorney General guidelines for preparing and conducting photo and live lineup identification procedures. Trenton, NJ: New Jersey Attorney General's Office. Available at www.eyewitnessconsortium.utep.edu/Documents/photoid.pdf.

LiNDSAY, R. C. L. (1999). Applying applied research: Selling the sequential line-up. Applied Cognitive Psychology, 13, 219-225.

LiNDSAY, R. C. L., \& WeLlS, G. L. (1985). Improving eyewitness identifications from lineups: Simultaneous versus sequential lineup presentation. Journal of Applied Psychology, 70, 556-564.

MacLin, O. H., \& MaLPass, R. S. (2005). Eyewitness identification: Information gain in a fair comparison of sequential and simultaneous lineups. Manuscript in preparation, University of Northern Iowa.

Maclin, O. H., \& Meissner, C. A. (2005). Project Waterloo: Developing a computerized system of lineup construction and administration for law enforcement. Manuscript in preparation, University of Northern Iowa.

MacLin, O. H., Meissner, C. A., \& Zimmerman, L. A. (2005). PC_Eyewitness: A computerized framework for the administration and practical application of research in eyewitness psychology. Behavior Research Methods, 37, 324-334.

MacLin, O. H., Zimmerman, L. A., \& Malpass, R. S. (2005). PC_Eyewitness and the sequential superiority effect: Computer-based lineup administration. Law \& Human Behavior, 29, 303-321.

Meissner, C. A., Tredoux, C. G., Parker, J. F., \& Maclin, O. H. (2005). Eyewitness decisions in simultaneous and sequential lineups: A dual-process signal detection theory analysis. Memory \& Cognition, 33, 783-792.

Scheck, B., Neufeld, P., \& Dwyer, J. (2000). Actual innocence. Garden City, NY: Doubleday.

Shull, R. L., \& PliskofF, S. S. (1967). Changeover delay and concurrent schedules: Some effects on relative performance measures. Journal of the Experimental Analysis of Behavior, 10, 517-527.

Steblay, N., Dysart, J., Fulero, S., \& Lindsay, R. C. L. (2001). Eyewitness accuracy rates in sequential and simultaneous lineup presentations: A meta-analytic comparison. Law \& Human Behavior, 25, 459-473.

TAslitz, A. (2004). Report to House of Delegates. American Bar Association, Criminal Justice Section. Available at www.atjsupport.org/ Defender/forensics/for_lib/Documents/1101916941.79/ABA\%20 report $\% 20111 \mathrm{c} \% 20$ adobe.pdf.

Technical Working Group for Eyewitness Evidence (2003). Eyewitness evidence: A trainer's manual for law enforcement. Washington, DC: National Institute of Justice.

Turtle, J., \& Steblay, N. (2005, March). Lineup identification issues with real officers and real cases: Addressing legal, logistical, and lamentable problems. Paper presented at the American PsychologyLaw Society Conference, La Jolla, CA.

WeLLs, G. L. (1984). The psychology of lineup identifications. Journal of Applied Social Psychology, 14, 89-103.

WELLS, G. L. (1993). What do we know about eyewitness identification? American Psychologist, 48, 553-557.

Wells, G. L., Malpass, R. S., Lindsay, R. C. L., Fisher, R. P., TurTLE, J. W., \& Fulero, S. M. (2000). From lab to the police station: A successful application of eyewitness research. American Psychologist, 55, 581-598.

Wells, G. L., Small, M., Malpass, R. S., Penrod, S., Fulero, S., \& BRIMACombe, C. A. E. (1998). Eyewitness identification procedures: Recommendations for lineups and photospreads. Law \& Human Behavior, 23, 603-647.

Wells, G. L., \& Windschitl, P. D. (1999). Stimulus sampling and social psychological experimentation. Personality \& Social Psychology Bulletin, 25, 1115-1125.

(Manuscript received March 14, 2006; revision accepted for publication September 12, 2006.) 